\title{
Approximating Minimum Independent Dominating Sets in Wireless Networks
}

\author{
Johann L. Hurink, Tim Nieberg \\ University of Twente \\ Faculty of Electrical Engineering, Mathematics \& Computer Science \\ Postbus 217, NL-7500 AE Enschede \\ $\{j .1$. hurink; t.nieberg\}@utwente.nl
}

\begin{abstract}
We present the first polynomial-time approximation scheme (PTAS) for the Minimum Independent Dominating Set problem in graphs of polynomially bounded growth. Graphs of bounded growth are used to characterize wireless communication networks, and this class of graph includes many models known from the literature, e.g. (Quasi) Unit Disk Graphs. An independent dominating set is a dominating set in a graph that is also independent. It thus combines the advantages of both structures, and there are many applications that rely on these two structures e.g. in the area of wireless ad hoc networks.

The presented approach yields a robust algorithm, that is, the algorithm accepts any undirected graph as input, and returns a $(1+\varepsilon)$-approximate minimum dominating set, or a certificate showing that the input graph does not reflect a wireless network.

Keywords: Minimum Independent Dominating Set, Minimum Maximal Independent Set, PTAS, Wireless Communication, Bounded Growth
\end{abstract}

\section{Introduction}

This paper presents and discusses a Polynomial-Time Approximation Scheme (PTAS) for the Minimum Independent Dominating Set problem in wireless communication networks.

A subset of vertices in a graph is independent if no two vertices are connected by an edge. A subset of vertices is called dominating if every vertex in the graph is either in the subset, or adjacent to a vertex from the subset. We are looking at the problem of finding a subset of vertices of small cardinality that is both independent and dominating in a graph that has a structure stemming from a wireless communication topology.

From an application point of view, independent and dominating set in a communication network are important structures, and many optimization approaches rely on these. 
In clustering schemes, independent sets result in clusterheads that have local control of their cluster without interference. Additionally, a dominating independent set based clustering scheme ensures that the entire network is covered. To this end, there are numerous clustering schemes based on such a structure in the literature. For example, especially in energy-efficient wireless computing, clustering allows for some wireless nodes to perform less tasks by delegating them to their respective clusterhead. On the other hand, the tasks of these clusterheads then result in additional energy consumption. Here, using as few clusterheads as possible, i.e. choosing them according to a minimum independent dominating set, results in energy savings for the network [7].

Next, we discuss some related work, followed by definitions and characterizations of wireless communication graphs in Section 2. In Section 3, we present the algorithm that yields a PTAS for the Minimum Independent Dominating Set problem in graphs that model wireless networks. In Section 4, we discuss the result obtained, and also show how to modify the algorithm towards a robust approach. The paper concludes in Chapter 5.

\subsection{Related Work}

Most of the work on optimization algorithms for and in wireless networks has been done using geometric graphs as underlying models for the communication network. Here, Unit Disk Graphs (UDG) are probably the most prominent class of graphs used [12]. These are defined as the intersection graph of equal diameter disks in the Euclidean plane. In [4], it is shown that many optimization problems, including the Minimum Dominating and Maximum Independent Set problems, remain $N P$-hard even when restricting the input to this class of graphs.

An important detail when using UDGs is the encoding of the input instance. Basically, there are two ways of describing a geometric intersection graph: by its adjacency and by its geometric information. While the first presents the graph as an undirected graph, the latter conveys more information that can be exploited explicitely by respective algorithms. Note that this is a significant distinction because determining for a given graph whether it is a disk graph is an NP-complete problem $[1,10]$, and therefore computing feasible positions to each node in a disk graph is also intractable.

In case geometric information is available, we can use geometric separation and a shifting strategy to obtain a PTAS for many problems on (Unit) Disk Graphs. This strategy gives a PTAS for the Maximum Independent Set, Minimum Dominating Set, and Minimum Vertex Cover problems on UDGs [2, 9], and the Minimum Connected Dominating Set problem on UDGs [3]. Combined with a dynamic programming approach, the shifting strategy also gives a PTAS for the Maximum Independent Set problem on Disk Graphs with arbitrary radii $[6]$.

To the best of our knowledge, even for the case of Unit Disk Graphs with given representation, a PTAS for the MIN-IDS problem is not known in the literature [5]. 
Without geometric information, and for the significantly larger class of graphs with polynomially bounded growth, robust approximation schemes for the Maximum Independent Set problem [16] and the Minimum Dominating Set problem [15] exist. For graphs of (polynomially) bounded growth, any maximal independent set, e.g. created by a simple greedy strategy, yields a constant-factor approximative solution to both the Maximum Independent Set and the Minimum Dominating Set problems [13]. It is easy to see that this solution then also gives a feasible solution to the MIN-IDS problem, together with a constant bound on the approximation guarantee. On a general graph, a Minimum Independent Dominating Set cannot be approximated within $n^{1-\varepsilon}$ for any $\varepsilon>0$ [8].

\section{Preliminaries}

Generally speaking, a communication network is modeled as an undirected graph $G=$ $(V, E)$, where the vertices $V$ represent the $n:=|V|$ devices or nodes, and two nodes are connected if they can communicate directly with one another. Consequently, a wireless network is created by the communication links between a collection of radio transceivers.

Two vertices of a graph are called independent if they are not adjacent to one another. A subset $I \subseteq V$ is called independent if all vertices are not connected. A subset $D \subseteq V$ is called dominating if every vertex from $V$ is contained in this subset, or adjacent to a vertex from $D$.

A subset can be both independent and dominating. Such a set is also called maximal independent set. Formally, an independent set is called maximal if it cannot be extended by the addition of any other vertex from the graph without violating the independence property. It is easy to verify that a maximal independent set is dominating.

If we seek for a small subset of vertices that is both dominating and independent, we call the resulting problem Minimum Independent Dominating Set problem (MIN-IDS). Since any maximal independent set also dominates a graph, the Min-IDS problem is sometimes also referred to as Minimum Maximal Independent Set problem.

Furthermore, we would like to point out that the Maximum Independent Dominating Set problem is equivalent to finding a Maximum Independent Set, which has to be maximal, and thus also dominating.

An algorithm that runs in polynomial time, and that for a given problem, always returns a feasible solution of relative error no more than some $\alpha \geq 1$ is called $\alpha$-approximation. A Polynomial-Time Approximation Scheme (PTAS) is an algorithm which, in addition to an input instance, requires a parameter $\varepsilon>0$, and which then returns a feasible solution with an approximation ratio of $1+\varepsilon$. The running time of such algorithms is allowed to depend on $\varepsilon$, but should be polynomial for fixed $\varepsilon>0$. In our case, a PTAS for the Min-IDS problem returns an independent and dominating set of cardinality at most $(1+\varepsilon)$ times the cardinality of a minimum independent and dominating set. 
We denote by $\Gamma(v)$ the closed neighborhood of a vertex $v \in V$, i.e. $\Gamma(v):=\{u \in V \mid$ $(u, v) \in E\} \cup\{v\}$. Analogously, for $V^{\prime} \subseteq V$, let $\Gamma\left(V^{\prime}\right):=\bigcup_{v \in V^{\prime}} \Gamma(v)$. For $r \in \mathbb{N}$, we call $\Gamma_{r}(v):=\Gamma\left(\Gamma_{r-1}(v)\right)$ the recursively defined $r$-th neighborhood of $v \in V$, with $\Gamma_{0}(v):=\{v\}$.

\subsection{Wireless Communication Graphs}

The nature of wireless transmissions leads to a structured graph. We now introduce the class of graphs of polynomially bounded growth to characterize wireless communication networks.

Definition 2.1 Let $G=(V, E)$ be a graph. If there exists a function $f($.$) such that ev-$ ery $r$-neighborhood in $G$ contains at most $f(r)$ independent vertices, then $G$ is $f$-growthbounded. Furthermore, we say that $G$ has polynomially bounded growth if for some constant $c \geq 1, f(r)$ is bounded by a polynomial of maximal degree c, i.e. $f(r)=O\left(r^{c}\right)$.

Note that the growth function $f($.$) only depends on the radius of the neighborhood, and not$ on the number of vertices in $G$. Thus, for constant $r$, the number of independent vertices in $\Gamma_{r}(v)$ is bounded by a constant for any $v \in V$. It is straightforward to verify that the bounded growth property of a graph is closed under taking vertex-induced subgraphs.

The above structure of polynomially bounded growth captures the major graph classes used to model wireless networks, including Unit Disk Graphs [4], Quasi Disk Graphs [11], and - leaving the disk shape behind - Coverage Area Graphs [14]. For example, the growth polynomial of a Unit Disk Graph is $p_{\mathrm{UDG}}(r)=(2 r+1)^{2}$. This can easily be seen by a geometric packing argumentation. Furthermore, it is $p_{\mathrm{UDG}}(1)=5$, since such a graph is $K_{1,6}$-free. Nevertheless, while most existing wireless graph models rely on geometric information of the nodes and their transmission ranges, bounded growth is defined independent of any geometric information.

\section{$2.2 \quad$ Neighborhoods and partial solutions}

For local neighborhoods, and subsets of the vertices in general, we now define local or partial solutions. Let $\mathcal{P}(V)$ denote the set of all subsets of vertices in $G$, and let $D: \mathcal{P}(V) \rightarrow \mathcal{P}$ be a function that returns for a set $V^{\prime}$ a minimum cardinality independent dominating set in $G$. In the following, we denote by $D^{*}$ an optimal solution to the MiN-IDS problem on $G$, in other words, $D^{*}:=D(V)$.

The function $D($.$) is always computed with respect to the entire underlying graph$ $G=(V, E)$. It may thus include vertices from outside the argument subset in its returned solution, i.e. , for a subset $V^{\prime} \subset V$ the inclusion $D\left(V^{\prime}\right) \subseteq V^{\prime}$ needs not to hold. This can be seen in the example given in Figure 1, where the black vertices represent an optimal independent dominating set of the 2-neighborhood of $v$, and $u \notin \Gamma_{2}(v)$. On the other hand, it is easy to see that $D\left(V^{\prime}\right) \subseteq \Gamma\left(V^{\prime}\right)$ always holds. 


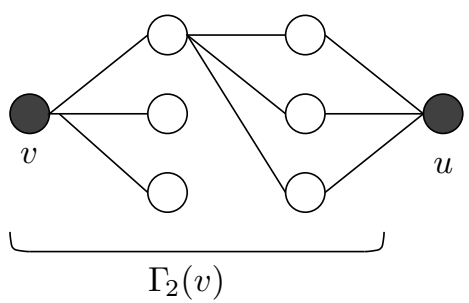

Figure 1: Example of a local solution $D\left(\Gamma_{2}(v)\right)=\{u, v\}$.

In order to improve readability, for neighborhoods of bounded radius, we introduce $D_{r}(v):=D\left(\Gamma_{r}(v)\right)$ as shorthand notation. If the central vertex of a neighborhood or a local solution is unambiguous, we omit it in the following for better readability, e.g. $D_{r}:=D_{r}(v)=D\left(\Gamma_{r}(v)\right)$ and $\Gamma_{r}:=\Gamma_{r}(v)$.

\subsection{Computing and bounding local solutions}

Let us now consider a graph $G=(V, E)$ with polynomially bounded growth, and let $p$ denote the polynomial growth function. Let $I_{r} \subseteq \Gamma_{r}$ denote a maximal independent set in this neighborhood. Then, $I_{r}$ also dominates $\Gamma_{r}$, and by the definition of bounded growth, we obtain

$$
\left|D\left(\Gamma_{r}\right)\right| \leq\left|I_{r}\right| \leq p(r) .
$$

Using this bound on the cardinality of the locally optimal solutions, it becomes clear that we can obtain an optimal solution $D_{r}$ for this neighborhood $\Gamma_{r}$ in polynomial time if the radius $r$ is bounded.

Lemma 2.2 Let $G=(V, E)$ be of polynomially p-bounded growth. For any neighborhood $\Gamma_{r}$, we can construct $D_{r}$ in time $n^{O(p(r))}$.

Further on, we will also need to describe the local neighborhoods and their distance with respect to each other by the following definition.

Definition 2.3 For a graph $G=(V, E)$, let $\mathcal{S}=\left\{S_{1}, \ldots, S_{k}\right\}$ be a collection of subsets of vertices $S_{i} \subseteq V, i=1, \ldots, k$, with the following property: for any two vertices $s \in S_{i}$ and $\bar{s} \in S_{j}, i \neq j$,

$$
d(s, \bar{s})>d
$$

holds. We refer to $\mathcal{S}$ as a $\boldsymbol{d}$-separated collection of subsets.

Clearly, for $d \geq 0$, the subsets of a $d$-separated collection are mutually disjoint. With respect to the Min-IDS problem, we get the following lower bound for a $d$-separated collection of subsets, $d \geq 2$, and independent dominating sets for the respective subsets. 
Lemma 2.4 For a d-separated collection $\mathcal{S}=\left\{S_{1}, \ldots, S_{k}\right\}$ in a graph $G=(V, E)$, with $d \geq 2$, it is

$$
\left|D^{*}\right| \geq \sum_{i=1}^{k}\left|D\left(S_{i}\right)\right| .
$$

For each subset $S_{i} \in \mathcal{S}$, consider the neighborhood $\Gamma\left(S_{i}\right)$. By definition, these are pairwise disjoint, and thus we get

$$
\left|D^{*}\right| \geq \sum_{i=1}^{k}\left|D^{*} \cap \Gamma\left(S_{i}\right)\right| .
$$

Furthermore, any vertex outside $\Gamma\left(S_{i}\right)$ has distance $d \geq 2$ to all vertices in $S_{i}$. Thus, $D^{*} \cap \Gamma\left(S_{i}\right)$ has to dominate $S_{i}$, and is independent in $G$.

Also, the set $D\left(S_{i}\right) \subset \Gamma\left(S_{i}\right)$ is independent and dominates $S_{i}$ in $G$. However, since $D\left(S_{i}\right)$ uses a minimum number of vertices to do so, we obtain $\left|D^{*} \cap \Gamma\left(S_{i}\right)\right| \geq\left|D\left(S_{i}\right)\right|$, and the claim follows.

Note that, in the above lemma, the overall union of the partial dominating sets $D\left(S_{i}\right), i=$ $1, \ldots, k$, needs not be an independent set. For example, consider the graph given in Figure 1, and suppose that we have two copies of it, where the rightmost vertices $u$ of each copy are connected. The two neighborhoods $\Gamma_{2}(v)$ are 2 -separated, while the union of the two partial dominating sets $D\left(\Gamma_{2}(v)\right)$ does not satisfy the independence property.

Enlarging the subsets of a $d$-separated collection, while preserving some bound on the cardinality of the partial solutions, we immediately obtain the following upper bound.

Corollary 2.5 Let $\mathcal{S}=\left\{S_{1}, \ldots, S_{k}\right\}$ be a d-separated collection in $G=(V, E), d \geq 2$, and let $T_{1}, \ldots, T_{k}$ be subsets of $V$ with $S_{i} \subseteq T_{i}$ for all $i=1, \ldots, k$. If there exists a bound $\rho \geq 1$ such that $\left|D\left(T_{i}\right)\right| \leq \rho \cdot\left|D\left(S_{i}\right)\right|$ holds for all $i=1, \ldots, k$, then

$$
\left|\bigcup_{i=1}^{k} D\left(T_{i}\right)\right| \leq \rho \cdot\left|D^{*}\right| .
$$

$\left|\bigcup_{i=1}^{k} D\left(T_{i}\right)\right| \leq \sum_{i=1}^{k}\left|D\left(T_{i}\right)\right| \leq \rho \cdot \sum_{i=1}^{k}\left|D\left(S_{i}\right)\right| \leq \rho \cdot\left|D^{*}\right|$.

In the approach described in the next section, we apply the above observation to local neighborhoods, and optimal solutions for these.

\section{Approximation algorithm}

In this section, we present the algorithm that yields a PTAS for the MIN-IDS problem on polynomially bounded growth graphs. The approach works in two stages. The first part of the approach follows [15], where this approach yields a PTAS for the Minimum Dominating Set problem. However, the solution set $\bar{D}$ returned by this algorithm needs 


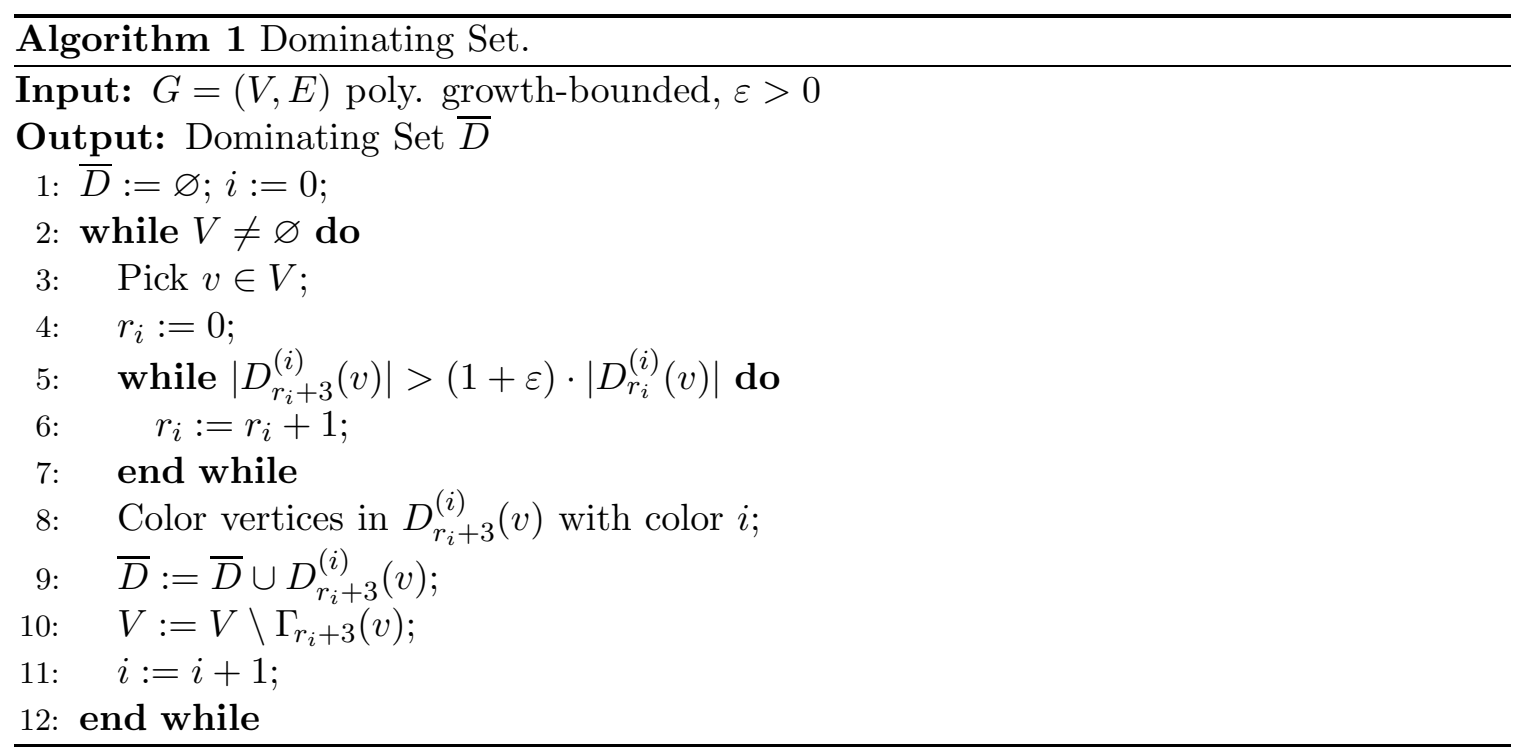

not be an independent set. The second stage then consists of a repair algorithm that restores the independence property without increasing the solution set too much.

\subsection{Local independent dominating sets}

Consider Algorithm 1, the main part of it iteratively constructs independent dominating sets for the neighborhoods $\Gamma_{r}$, and stops increasing the radius $r$ of the neighborhoods if

$$
\left|D_{r+3}\right| \leq(1+\varepsilon) \cdot\left|D_{r}\right|
$$

holds. Then, the solution $\bar{D}$ and the set of remaining vertices is updated respectively. In this context, we call $D_{r+3}^{(i)}$ a partial solution, and the color $i$ also gives the iteration.

It is easy to see that the set $\bar{D}$ dominates the entire graph $G$ since at each iteration $i$, the removed neighborhood $\Gamma_{r_{i}+3}(v)$ is dominated by $D_{r_{i}+3}^{(i)}(v)$, which is added to the solution set. Furthermore, the radius of the largest neighborhood we need to consider is bounded by a constant that only depends on the growth function and $\varepsilon>0$, and especially not on the size of the graph, as follows.

Lemma 3.1 Let $G=(V, E)$ be a graph of polynomially p-bounded growth. There exists a constant $c=c(\varepsilon)$ such that $r_{i}<c$ for every $i$.

Recall that $r_{i}$ denotes the radius of the neighborhood considered when the criterion to stop expanding a neighborhood is met. Now, suppose that $r<r_{i}$ is divisible by three, in this case we have

$$
\left.p(r+3) \geq\left|D_{r+3}\right|>(1+\varepsilon) \cdot \mid D_{r}\right)\left|>\cdots>(1+\varepsilon)^{r / 3} \cdot\right| D_{0} \mid=(\sqrt[3]{1+\varepsilon})^{r},
$$




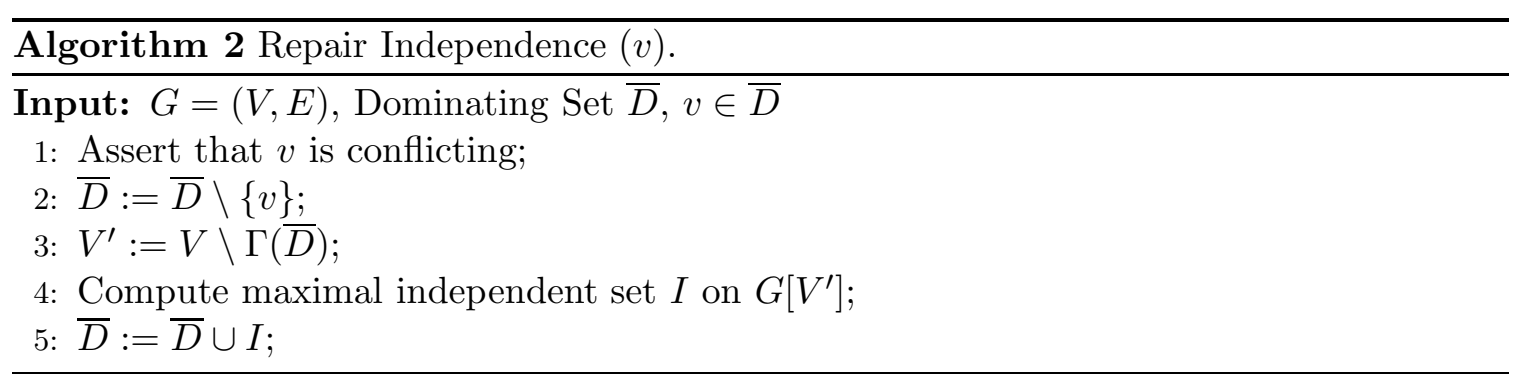

which eventually has to be violated. The other two cases follow the same argumentation.

Due to Corollary 2.5, we see that $\bar{D}$ satisfies the inequality $|\bar{D}| \leq(1+\varepsilon)\left|D^{*}\right|$. However, $\bar{D}$ may not be an independent set, and we resolve this issue next.

\subsection{Restoring global independence}

Consider the graph $G[\bar{D}]$ induced by the dominating set $\bar{D}$. If there are still edges present in $G[\bar{D}]$, we resort to Algorithm 2, which removes non-independent vertices, and adds independent ones as follows. Vertices in $\bar{D}$ that are not independent are called conflicting vertices.

Let $v \in \bar{D}$ be a conflicting vertex, we then solve the conflicts by removing $v$ and restoring domination again by greedy addition of vertices that are independent with respect to the remaining ones in $\bar{D}$.

Lemma 3.2 Algorithm 2 removes all conflicts involving the argument vertex $v$, and does not introduce any new conflicting vertices into $\bar{D}$.

Clearly, removing $v$ from $\bar{D}$ removes respective conflicts. The set $V^{\prime}$, by construction, is 1-separated to $\bar{D}$ in $G$, and we can thus add a maximal independent set $I \subset V^{\prime}$ to $\bar{D}$ that is not adjacent to $\bar{D}$. Since $I$ dominates $V^{\prime}$, the overall set $\bar{D} \cup I$ dominates $V$ in $G$.

As for the new vertices added to $\bar{D}$ at each invocation of Algorithm 2, we can bound the number of additions as follows.

Lemma 3.3 Let $G=(V, E)$ be a graph of p-bounded growth. A single invocation of Algorithm 2 increases the cardinality of $\bar{D}$ by at most $p(1)$.

It is easy to see that $V^{\prime} \subset \Gamma(v)$ holds, otherwise $\bar{D}$ is not a dominating set. Any independent set in $\Gamma(v)$ consists of at most $p(1)$ vertices due to the bounded growth property of $G$, and therefore $|I| \leq p(1)$.

In order to repair a solution, we create a candidate set $C \subset \bar{D}$ by adding conflicting vertices according to their color as follows. Initially, start with $C$ being empty. Consider 
the first iteration, i.e. $D_{r_{0}+3}^{(0)}$ denotes the independent dominating set constructed in this iteration. Looking at the overall constructed solution $\bar{D}$, we add all conflicting vertices from $D_{r_{0}+3}^{(0)}$ to $C$. We then proceed iteratively until all colors have been accounted for. Note that in each iteration we only need to consider conflicting vertices involving higher colors. It is straightforward to verify that $\bar{D} \backslash C$ is an independent set in $G$ after all partial solutions have been considered.

As for the cardinality of $C$, we have the following observation.

Observation $3.4|C| \leq \varepsilon \cdot\left|D^{*}\right|$.

In order to prove the observation, we proceed alongside the colors given to the vertices in Algorithm 1. Consider the local neighborhoods $\Gamma_{r_{0}}$ and independent dominating sets $D_{r}^{(0)}$ of the first iteration. Then, $D_{r_{0}+3}^{(0)}$ is the partial solution of this iteration such that

$$
\left|D_{r_{0}+3}^{(0)}\right| \leq(1+\varepsilon) \cdot\left|D_{r_{0}}^{(0)}\right| \cdot
$$

We see that any conflicting vertex with color 0 has to be outside of $\Gamma_{r_{0}+1}=\Gamma\left(\Gamma_{r_{0}}\right)$, that is,

$$
C \cap D_{r_{0}+3}^{(0)} \subseteq D_{r_{0}+3}^{(0)} \backslash \Gamma_{r_{0}+1}
$$

holds. On the other hand, the interior part $D_{r_{0}+3}^{(0)} \cap \Gamma_{r_{0}+1}$ dominates the neighborhood $\Gamma_{r_{0}}$, and therefore

$$
\left|D_{r_{0}+3}^{(0)} \cap \Gamma_{r_{0}+1}\right| \geq\left|D_{r_{0}}^{(0)}\right|,
$$

as $D_{r_{0}}^{(0)}$ is an optimal independent dominating set for the same neighborhood.

Partitioning $D_{r_{0}+3}^{(0)}$ now yields

$$
\left|D_{r_{0}+3}^{(0)}\right|=\left|D_{r_{0}+3}^{(0)} \backslash \Gamma_{r_{0}+1}\right|+\left|D_{r_{0}+3}^{(0)} \cap \Gamma_{r_{0}+1}\right| \leq(1+\varepsilon) \cdot\left|D_{r_{0}}^{(0)}\right|,
$$

and we can conclude that

$$
\left|C \cap D_{r_{0}+3}^{(0)}\right| \leq\left|D_{r_{0}+3}^{(0)} \cap \Gamma_{r_{0}+1}\right| \leq \varepsilon \cdot\left|D_{r_{0}}^{(0)}\right|
$$

has to hold.

We continue with the same argumentation for the local subsets of the succeeding colors. Denote by $C_{i}$ the set of remaining conflicting vertices after the removal of vertices with colors lower than $i$. Keeping in mind that vertices with lower color no longer induce conflicts, we see that for every iteration $i$, we obtain

$$
\left|C_{i} \cap D_{r_{i}+3}^{(i)}\right| \leq \mid D_{r_{i}+3}^{(i)} \backslash \Gamma\left(\Gamma_{r_{i}}|\leq \varepsilon \cdot| D_{r_{i}}^{(i)} \mid .\right.
$$


Let $i_{\max }$ denote the last iteration of of the first stage of the approximation Algorithm 1, which is then also the highest color used. Using the fact that $\left\{D_{r_{0}}^{(0)}, \ldots, D_{r_{i_{\max }}}^{\left(i_{\max }\right)}\right\}$ is a 1-separated collection of partially optimal subsets, we get

$$
|C| \leq \sum_{i=1}^{i_{\max }}\left|D_{r_{i}+3}^{(i)} \backslash \Gamma\left(\Gamma_{r_{i}}\right)\right| \leq \varepsilon \cdot \sum_{i=1}^{i_{\max }}\left|D_{r_{i}}^{(i)}\right| \leq \varepsilon \cdot\left|D^{*}\right|,
$$

as claimed.

After having repaired all conflicts in $C$, the overall independent dominating set $D$ constructed by the approach is then of cardinality

$$
|D| \leq|\bar{D}|+(p(1)-1) \cdot|C| \leq(1+p(1) \cdot \varepsilon) \cdot\left|D^{*}\right|
$$

again where $\bar{D}$ denotes the $(1+\varepsilon)$-approximate dominating set returned by the first stage of the approach, and $D^{*}$ gives an optimal solution.

The overall run time of the algorithm is dominated by the dominating set construction in Algorithm 1. The time complexity of this algorithm is $n^{O\left(\frac{1}{\varepsilon} \log \frac{1}{\varepsilon}\right)}$ for $\varepsilon>0$, see [15].

\section{Discussion}

We now present a simple way to make the above approximation schemes robust. In this case, the robust algorithm accepts any undirected graph as valid input, and either returns a desired approximate solution, or outputs a polynomial certificate showing that the input graph does not satisfy the structural assumption of $p$-bounded growth [17].

In the previous section, we have seen that the approximation algorithm actually yield a PTAS when the instance reflects a graph of polynomially $p$-bounded growth. We thus continue the discussion only for the case that the undirected graph $G=(V, E)$ presented to the algorithm does not satisfy the characterization of a polynomially bounded growth graph.

Observe that during the first stage of the approximation, the polynomial runtime of the approximation algorithms results from the bound $p(r)$ on the size of an independent set, i.e. $\left|D_{r}\right| \leq p(r)$. If, during execution of the algorithm, a neighborhood $\Gamma_{r}$ contains an independent set of size larger than $p(r)$, we can use this neighborhood as a polynomial certificate showing non-membership in the class of $p$-Bounded Growth Graphs. An independent dominating set that gives an upper bound on the size of $D_{r}$ can be quickly computed by a greedy strategy, and if this independent set satisfies the bound $p(r)$, we can continue without problems.

In the second stage, locally verifying that the size of the independent set added while resolving conflicts meets the $p(1)$ bound is straightforward. 
Generally speaking, we can thus apply the approximation scheme to any undirected graph which is believed to be of polynomially bounded growth, without risk of failure, i.e. exponential running time, if this assumption is wrong. In wireless networks, this gives the advantage that we can indirectly account for all the uncertainties and the dynamic behavior which govern the resulting applications: there may exist wireless communication graphs in very harsh environments for which the polynomially bounded growth assumption does not hold. In this case, we at least receive according feedback.

We summarize with the following theorem.

Theorem 4.1 There exists a PTAS for the MIN-IDS problem on polynomially growth bounded graphs. In addition to the desired approximation guarantee, this PTAS requires only adjacency information of the input graph. Furthermore, the algorithms of the scheme are robust.

\section{Conclusion}

In this paper, we looked at the Minimum Independent Dominating Set problem in wireless communication networks. We present a polynomial-time approximation scheme for this problem on graph with polynomially bounded growth. The scheme is robust and thus returns for any undirected graph given as input a meaningful output.

The approximation algorithm that results in the PTAS works by exploiting the fact that the graph can be divided into local neighborhoods, for which an optimal, partial solution can be obtained. While this approach is already used for related problems, feasibility when combining the partial solutions is an issue for sets that have to remain both independent and dominating. We solve this issue by a post-processing repair algorithm.

The run time of the $(1+\varepsilon)$-approximation that gives the PTAS is $n^{O\left(\frac{1}{\varepsilon} \log \frac{1}{\varepsilon}\right)}$ for $\varepsilon>0$.

\section{References}

[1] H. Breu and D.G. Kirkpatrick. Unit disk graph recognition is NP-hard. Computational Geometry. Theory and Applications, 9(1-2):3-24, 1998.

[2] T.M. Chan. Polynomial-time approximation schemes for packing and piercing fat objects. Journal of Algorithms, 46(2):178-189, 2003.

[3] X. Cheng, X. Huang, D. Li, W. Wu, and D.-Z. Du. A polynomial-time approximation scheme for the minimum-connected dominating set in ad hoc wireless networks. Networks, 42:202-208, 2003.

[4] B. N. Clark, C. J. Colburn, and D. S. Johnson. Unit disks graphs. Discrete Mathematics, $86: 165-177,1990$. 
[5] P. Crescenzi and V. Kann (Eds.). A compendium of NP optimization problems. http://www.nada.kth.se/ viggo/problemlist/.

[6] T. Erlebach, K. Jansen, and E. Seidel. Polynomial-time approximation schemes for geometric intersection graphs. SIAM Journal of Computing, 34(6):1302-1323, 2005.

[7] EYES. Energy-Efficient Sensor Networks. (IST-2001-34734), http://www.eyes.eu.org.

[8] M.M. Halldorson. Approximating the minimum maximal independence number. Information Processing Letters, 46:169-172, 1993.

[9] H.B. Hunt III, M.V. Marathe, V. Radhakrishnan, S. S. Ravi, D.J. Rosenkrantz, and R.E. Stearns. NC-approximation schemes for NP- and PSPACE-hard problems for geometric graphs. Journal of Algorithms, 26(2):238-274, 1998.

[10] J. Kratochvil. Intersection graphs of noncrossing arc-connected sets in the plane. In Proc. Symp. on Graph Drawing (GD'96), pages 257-270, 1997.

[11] F. Kuhn, R. Wattenhofer, and A. Zollinger. Ad-hoc networks beyond unit disk graphs. In Proc. 1st ACM DIALM-POMC Joint Workshop on Foundations of Mobile Computing, 2003.

[12] X.Y. Li. Algorithmic, geometric and graphs issues in wireless networks. Wireless Communications and Mobile Computing, 3:119-140, 2003.

[13] M.V. Marathe, H. Breu, H.B. Hunt III, S. S. Ravi, and D.J. Rosenkrantz. Simple heuristics for unit disk graphs. Networks, 25:59-68, 1995.

[14] T. Nieberg and J.L. Hurink. Wireless communication graphs. In Proc. Intelligent Sensors, Sensor Networks and Information Processing Conference, pages 367-372, 2004.

[15] T. Nieberg and J.L. Hurink. A PTAS for for the minimum dominating set problem in unit disk graphs. In Proc. 3rd workshop on approximation and online algorithms, pages 296-306. Springer, LNCS 3879, 2005.

[16] T. Nieberg, J.L. Hurink, and W. Kern. A robust PTAS for maximum independent sets in unit disk graphs. In Proc. 30th workshop on graph theoretic concepts in computer science, pages 214-221. Springer, LNCS 3353, 2004.

[17] V. Raghavan and J. Spinrad. Robust algorithms for restricted domains. Journal of Algorithms, 48(1):160-172, 2003. 